\title{
Birth outcomes and pregnancy complications of women with uterine leiomyoma-a population-based case-control study
}

\author{
Ferenc Bánhidy ${ }^{1}$, Nándor Ács ${ }^{1}$, Erzsébet H. Puhó ${ }^{2}$, Andrew E. Czeizel ${ }^{2 *}$ \\ ${ }^{1}$ Second Department of Obstetrics and Gynecology, Semmelweis University, School of Medicine, Budapest, Hungary \\ ${ }^{2}$ Foundation for the Community Control of Hereditary Diseases, Budapest, Hungary; ${ }^{*}$ Corresponding Author: czeizel@interware.hu
}

Received 16 December 2009; revised 6 January 2010; accepted 7 January 2010.

\begin{abstract}
Objective Uterine leiomyoma is not a rare pathological condition in pregnant women; thus the aim of the study was to evaluate the recent progress in the treatment of these pregnant women on the basis of the association of leiomyoma in pregnancy (LP) with pregnancy complications and birth outcomes including structural birth defects, i.e. congenital abnormalities (CA) in the offspring. Design Cases with CA and matched controls without $C A$ in the population-based Hungarian Case-Control Surveillance System of Congenital Abnormalities (HCC SCA) were evaluated. Only women with prospectively and medically recorded LP in prenatal maternity logbook and medically recorded birth outcomes (gestational age, birth weight, CA) were included to the study. Setting the HCCSCA, 1980-1996 contained 22,843 cases with CA and 38,151 matched controls without CA. Population Hungarian pregnant women and their informative offspring: live births, stillbirths and prenatally diagnosed malformed fetuses. Methods Comparison of birth outcomes of cases with matched controls and pregnancy complications of pregnant women with or without LP. Main outcome measures Pregnancy complications, mean gestational age at delivery and birth weight, rate of preterm birth, low birthweight, CA. Results A total of $34(0.15 \%)$ cases had mothers with LP compared to $71(0.19 \%)$ controls. There was a higher incidence of threatened abortion, placental disorders, mainly abruption placentae and anaemia in mothers with LP. There was no significantly higher rate of preterm birth in the newborns of women with LP but their mean birth weight was higher and it
\end{abstract}

associated with a higher rate of large birthweight newborns. A higher risk of total CA was not found in cases born to mothers with LP (adjusted OR with $95 \% \mathrm{Cl}=0.7,0.5-1.1$ ), the specified groups of CAs were also assessed versus controls, but a higher occurrence of women with LP was not revealed in any CA group. Conclusions Women with LP have a higher risk of threatened abortion, placental disorders and anaemia, but a higher rate of adverse birth outcomes including CAs was not found in their offspring.

Keywords: Uterine Leiomyoma in Pregnant Women; Pregnancy Complications; Preterm Birth; Large Birth Weight; Congenital Abnormalities; Population-Based Case-Control Study

\section{INTRODUCTION}

Uterine leiomyoma (fibroid) is benign, smooth muscle tumour and most common non cancerous neoplasm in women of child-bearing age. Though the onset of uterine leiomyoma is increasing with advanced maternal age, this pathological condition occurs in pregnant women as well and because leiomyoma tends to grow under the influence of estrogens, $15-30 \%$ of leiomyoma may enlarge during the first trimester of pregnancy [1]. Compressive effect of leiomyoma may distort the intrauterine cavity and alter the endometrium thus after conception may interfere implantation, placental development and the growth of the conceptus mechanically [2]. In addition there is an increased uterine irritability and contractility secondary to rapid fibroid growth. Thus the direct mechanical effect and indirect alteration in oxytocinase activity may disrupt the normal progression of uterus and development of the fetus, therefore uterine leio- 
myoma is a cause of pregnancy loss, fetal malpresentation, intrauterine growth retardation and premature labour [3].

However, most studies of pregnancy complications and birth outcomes in uterine leiomyoma patients were composed of participants from only one hospital or clinic [4-7] and the results of population-based studies have been published only recently [8-9]. The objective of our study was the evaluation the possible association between maternal uterine leiomyoma in pregnancy (LP) and pregnancy complications, in addition adverse birth outcomes, particularly structural birth defects, i.e. congenital abnormalities (CAs) in the population-based data set of the Hungarian Case-Control Surveillance of Congenital Abnormalities (HCCSCA) [10].

\section{MATERIALS}

The protocol of the HCCSCA included five steps. The first step was the selection of cases from the data set of the Hungarian Congenital Abnormality Registry (HCAR), 1980-1996 [11] for the HCCSCA. Notification of CAs is compulsory for physicians from the birth until the end of first postnatal year to the HCAR. Most cases with CA are reported by obstetricians and paediatricians. In Hungary practically all deliveries take place in inpatient obstetric clinics and the birth attendants are obstetricians. Paediatricians are working in the neonatal units of inpatient obstetric clinics, or in various inpatient and outpatient paediatric clinics. Autopsy was mandatory for all infant deaths and common in stillborn fetuses during the study period. Pathologists sent a copy of the autopsy report to the HCAR if defects were identified in stillbirths and infant deaths. Since 1984 fetal defects diagnosed in prenatal diagnostic centres with or without termination of pregnancy have also been included into the HCAR. Isolated minor anomalies (e.g., umbilical hernia, small haemangioma, hydrocele) were recorded but not evaluated in the HCAR. The total (birth + fetal) prevalence of cases with CA diagnosed from the second trimester of pregnancy through the age of one year was 35 per 1000 informative offspring (liveborn infants, stillborn fetuses and electively terminated malformed fetuses) in the HCAR, 1980-1996, and about $90 \%$ of major CAs were recorded in the HCAR during the 17 years of the study period [12].

There were three exclusion criteria at the selection of cases with CAs from the HCAR for the data set of the HCCSCA. 1) Cases reported after three months of birth or pregnancy termination were excluded. The longer time between birth or pregnancy termination and data collection decreases the accuracy of information about pregnancy history. This group of excluding cases in- volved $33 \%$ of cases and most had mild CAs. 2) Three mild CAs (such as congenital dislocation of hip based on Ortolani click, congenital inguinal hernia, and large haemangioma), and 3) CA-syndromes caused by major mutant genes or chromosomal aberrations with preconceptional (i.e. non teratogenic) origin were also excluded.

The second step was to ascertain appropriate controls from the National Birth Registry of the Central Statistical Office for the HCCSCA. Controls were defined as newborn infants without CA. In most years two controls were matched to every case according to sex, birth week, and district of parents' residence.

\section{DATA COLLECTION AND EVALUATION}

The third step was to obtain the necessary maternal, particularly exposure data from three sources:

\subsection{Prospective Medically Recorded Data}

Mothers were asked in an explanatory letter to send us the prenatal maternity logbook and other medical records particularly discharge summaries concerning their diseases during the study pregnancy and their child's CA. Prenatal care was mandatory for pregnant women in Hungary (if somebody did not visit prenatal care clinic, she did not receive a maternity grant and leave), thus nearly $100 \%$ of pregnant women visited prenatal care clinics, on average 7 times in their pregnancies. The first visit was between the 6 th and 12 th gestational week. The task of obstetricians was to record all pregnancy complications, maternal diseases and related drug prescriptions in the prenatal maternity logbook.

\subsection{Retrospective Self-Reported Maternal Information}

A structured questionnaire along with a list of medicinal products (drugs and pregnancy supplements) and diseases, plus a printed informed consent form were also mailed to the mothers immediately after the selection of cases and controls. The questionnaire requested information on pregnancy complications and maternal diseases, on medicinal products taken during pregnancy according to gestational months, and on family history of CAs. To standardize the answers, mothers were asked to read the enclosed lists of medicinal products and diseases as a memory aid before they filled in the questionnaire. We also asked mothers to give a signature for informed consent form which permitted us to record the name and address of cases both in the HCCSCA and in the HCAR.

The mean \pm S.D. time elapsed between the birth or pregnancy termination and the return of the "information 
package" (questionnaire, logbook, discharge summary, and informed consent form) in our prepaid envelope was $3.5 \pm 1.2$ and $5.2 \pm 2.9$ months in the case and control groups, respectively

\subsection{Supplementary Data Collection}

Regional nurses were asked to visit all non-respondent case mothers at home and to help mothers to fill-in the same questionnaire used in the HCCSCA, to evaluate the available medical records, to obtain data regarding lifestyle (smoking, drinking, illicit drug use) through personal interview of mothers and her close relatives living together and to ask mothers to sign informed consent. Regional nurses visited only 200 non-respondent control and 600 other control mothers as part of two validation studies $[13,14]$ using the same methods as in non-respondent case mothers because the committee on ethics considered this follow-up to be disturbing to the parents of all healthy children.

Overall, the necessary information was available on $96.3 \%$ of cases $(84.4 \%$ from reply to the mailing, $11.9 \%$ from the nurse visit) and $83.0 \%$ of the controls $(81.3 \%$ from reply, $1.7 \%$ from visit). Informed consent form was signed by $98 \%$ of mothers, names and addresses were deleted in the rest $2 \%$.

The procedure of data collection in the HCCSCA was changed in 1997 such that regional nurses visited and questioned all cases and controls, however, these data have not been validated until now, thus only the data set of 17 years between 1980 and 1996 is evaluated here.

The fourth step at the evaluation of cases and controls in the HCCSCA is the definition of exposure and to determine its diagnostic criteria. The diagnosis of LP was based on the personal manual and ultrasound examination of pregnant women by obstetrician. In general the size of uterine leiomyoma and their types (intramural, subserosal, submucosal, pedunculated, etc) was given in the prenatal maternity logbook but unfortunately these data were not copied out. Pregnant women with dysfunctional uterine bleeding, endometrial polyp, endometriosis, etc were excluded from the study.

Gestational time was calculated from the first day of the last menstrual period. Beyond birth weight $(\mathrm{g})$ and gestational age at delivery (wk), the rate of low birthweight $(<2500 \mathrm{~g})$ and large birth weight $(4000$ or more g) newborns, in addition the rate of preterm births $(<37$ weeks) and postterm birth (42 or more weeks) were analyzed on the basis of discharge summaries of inpatient obstetric clinics. The critical period of most major CAs is in the second and/or third gestational month.

Drug treatments and folic acid/multivitamin supplements were also evaluated. The latter may indicate the level of pregnancy care, and indirectly may show the socio-economic status and the motivation of mothers to prepare and/or to achieve a healthy baby. In addition it is necessary to consider folic acid and folic acid-containing multivitamins in the evaluation of preventable CAs [15-18]. Other potential confounding factors included maternal age, birth order, marital and employment status which had a good correlation with the level of education and income, thus was regarded at the indicator of socioeconomic status [19], and high fever related diseases such as influenza.

We used SAS version 8.02 (SAS Institute Ins., Cary, North Carolina, USA) for statistical analyses as the fifth step of the HCCSCA. The occurrence of LP was compared in the two study groups and the crude odds ratios (OR) with 95\% confidence intervals (CI) were calculated. Contingency tables were prepared for the main study variables. The prevalence of other maternal diseases, drug intakes and pregnancy supplements used during pregnancy were compared between the group of case and control mothers with LP. We compared the prevalence of LP during the study pregnancy in specific CA groups including at least 2 cases with the frequency of LP in their all matched control pairs. Crude and adjusted OR with $95 \%$ CI were evaluated in conditional logistic regression models. We examined confounding variables by comparing the OR for LP in the models with and without inclusion of the potential confounding variables. Finally, maternal age $(<20 \mathrm{yr}, 20-29 \mathrm{yr}$, and $30 \mathrm{yr}$ or more), birth order (first delivery or one or more previous deliveries), employment status, influenzacommon cold (yes/no), and use of folic acid supplement (yes/no) were included in the models as potential confounders.

\section{RESULTS}

As it appeared at the preliminary evaluation of LP, two groups could be differentiated: 1) prospectively and medically recorded LP in the prenatal maternity logbook, and 2) LP based on retrospective maternal information in the questionnaire. However, the diagnosis of leiomyoma can be frequently questioned without medical record in the latter group and in general it was not possible to differentiate the leiomyoma with myomectomy before the study pregnancy. Thus, only the first group, i.e. medically recorded LP was evaluated.

The case group consisted of 22,843 malformed newborns or fetuses ("informative offspring"), of whom 39 $(0.15 \%)$ had mothers with medically recorded leiomyoma during the study pregnancy. However, of these 39 pregnant women, $5(12.8 \%)$ had previous myomectomy due to leiomyoma. The total number of births in Hungary was 2,146,574 during the study period between 
1980 and 1996. Thus the 38,151 controls represented $1.8 \%$ of all Hungarian births, and among those controls, 82 were born to mothers with medically recorded leiomyoma. Of these 82 control mothers, 11 (13.4\%) had previous myomectomy. Our objective was to evaluate the possible association of leiomyoma during the study pregnancy with pregnancy complications and adverse birth outcomes, therefore 34 case mothers $(0.15 \%)$ and 71 control mothers $(0.19 \%)$ with leiomyoma, i.e. LP were evaluated. Surgical intervention due to LP during the study pregnancy was not recorded in the prenatal maternity logbook and in the discharge summaries of these 105 pregnant women with LP. The number of pregnant women with previous myomectomy of leiomyoma was too small, thus these pregnant women were excluded from this analysis.

Of 34 case mothers, $30(88.2 \%)$, while of 71 control mothers, $60(84.5 \%)$ had diagnosed LP in the first visit of prenatal maternity clinic, thus the onset of this pathological condition was before conception. The so-called new-onset LP occurred in 4 case mothers and 11 control mothers diagnosed after the fourth gestational month.

Table 1 summarizes the characteristics of mothers with and without LP as reference. This comparison indicates a much higher mean maternal age (due to the larger proportion of women with the age group of 30 and more years) in women with LP. However, the mean birth order was only higher in control mothers with LP, but somewhat lower in case mothers with LP than case mothers without LP. Mean pregnancy order (previous birth + recorded miscarriages) was also evaluated, and the difference between birth and pregnancy order was somewhat higher in pregnant women with LP in both case mothers and control mothers and these data may indicate a higher rate of miscarriages in previous pregnancies. The proportion of unmarried pregnant women was larger in the groups of LP, while LP was more frequently recorded in the prenatal maternity logbooks of professional pregnant women. In the group of case mothers, the proportion of managerial women was also larger.

Among pregnancy supplements (Table 1), the use of folic acid and iron was similar between mothers with or without LP, but these supplements were used more frequently by control mothers. However, medicinal products containing calcium were used more frequently by mothers with LP, and a much higher rate of case mothers were treated with vitamin $\mathrm{E}$.

Of 2,640 case mothers visited at home, only 4 had LP and one was smoker during the study pregnancy. Of 2,636 mothers without LP, 576 (21.9\%) smoked. Of 800 control mothers visited at home, $152(19.0 \%)$ smoked during the study pregnancy.

Acute maternal diseases (e.g. influenza) did not occur more frequently in mothers with LP. Among chronic diseases, the prevalence of diabetes mellitus and epilepsy was similar in the study groups, but essential hypertension $(19.0 \%$ vs. $7.0 \%$, OR with $95 \%$ CI: 3.1 , $1.9-5.1)$, haemorrhoids ( $18.1 \%$ vs. $3.9 \%$, OR with $95 \%$ CI: $5.4,3.3-8.8)$ and constipation $(7.6 \%$ vs. $2.1 \%$, OR with 95\% CI: $3.9,1.9-8.1)$ were more frequent in 105 women with LP than in 60,889 mothers without LP.

The incidences of pregnancy complications are shown in Table 2, because they were different in case and control mothers with LP. Threatened abortion, placental disorders (mainly abruption placentae) and anaemia occurred more frequently in case mothers with LP than in case mothers without LP. However, LP did not associate with a higher rate of threatened abortion, placental disorders and anaemia in control mothers. Thus LP and fetal defects may have some causal association with the higher risk of certain pregnancy complications, such as placental disorders. Unexpectedly the incidence of threatened preterm delivery was not significantly higher in case and control mothers with LP.

There was some difference in the distribution and frequency of drugs used by mothers with LP explained by the higher use of antihypertensive (methyldopa, metoprolol, nifedipine) drugs (12.4\% vs. $2.6 \%)$ and the usual treatment of threatened abortion with allylestrenol $(21.0 \%$ vs. $14.5 \%)$ and diazepam $(25.7 \%$ vs. $11.3 \%)$ in Hungary. In addition the use of hydroxyprogesterone $(5.7 \%$ vs. $1.2 \%)$ and human chorionic gonadotropin $(2.9 \%$ vs. $0.3 \%)$ was more frequent in 105 pregnant women with LP than in 60,889 pregnant women without LP.

Birth outcomes are shown in case and control newborns (Table 3) but statistical testing was used only in controls because CAs may have a more drastic effect for these variables in cases than LP itself. (There was no difference in the sex ratio of the study groups, and twin did not occur among newborns of mothers with LP.) The mean gestational age at delivery was somewhat $(0.1 \mathrm{wk}$ in cases and $0.2 \mathrm{wk}$ in controls) longer, the rate of preterm birth was higher in controls but lower in cases. There was no difference in the rate of postterm births among study groups. However, these differences were not significant. The mean birth weight was 159 and $95 \mathrm{~g}$ larger in cases and controls of mothers with LP and these differences were significant. However, these differences were not reflected in the rate of low birthweight newborns because there was no difference in their rates between cases and controls born to mother with or without LP. There was a higher proportion of large birthweight newborns of both cases and controls but this difference was significant only in cases (OR with $95 \%$ CI: 3.0, 1.9-7.2). 
Table 1. Maternal characteristics of women with or without leiomyoma in pregnancy (LP).

\begin{tabular}{|c|c|c|c|c|c|c|c|c|}
\hline \multirow{4}{*}{$\begin{array}{c}\text { Variables } \\
\text { Quantitative }\end{array}$} & \multicolumn{4}{|c|}{ Case mothers } & \multicolumn{4}{|c|}{ Control mothers } \\
\hline & \multicolumn{2}{|c|}{ without } & \multicolumn{2}{|c|}{ with } & \multicolumn{2}{|c|}{ without } & \multicolumn{2}{|c|}{ with } \\
\hline & \multicolumn{4}{|c|}{ LP } & \multicolumn{4}{|c|}{ LP } \\
\hline & \multicolumn{2}{|c|}{$(\mathrm{N}=22,809)$} & \multicolumn{2}{|c|}{$(\mathrm{N}=34)$} & \multicolumn{2}{|c|}{$(\mathrm{N}=38,080)$} & \multicolumn{2}{|c|}{$(\mathrm{N}=71)$} \\
\hline Maternal age, yr. & No. & $\%$ & No. & $\%$ & No. & $\%$ & No. & $\%$ \\
\hline-19 & 2,506 & 11.0 & 0 & 0.0 & 3,277 & 8.6 & 0 & 0.0 \\
\hline $20-29$ & 15,580 & 68.3 & 13 & 38.2 & 25,777 & 72.4 & 25 & 35.2 \\
\hline $30-$ & 4,723 & 20.7 & 21 & 61.8 & 7,226 & 19.0 & 46 & 64.8 \\
\hline Mean, S.D. & \multicolumn{2}{|c|}{$25.5 \pm 5.3$} & \multicolumn{2}{|c|}{$32.1 \pm 6.0$} & \multicolumn{2}{|c|}{$25.4 \pm 4.9$} & \multicolumn{2}{|c|}{$31.9 \pm 5.7$} \\
\hline \multicolumn{9}{|l|}{ Birth order (parity) } \\
\hline 1 & 10,691 & 46.9 & 17 & 50.0 & 18,175 & 47.7 & 34 & 47.9 \\
\hline 2 or more & 12,118 & 53.1 & 17 & 50.0 & 19,905 & 52.3 & 37 & 52.1 \\
\hline Mean, S.D. & \multicolumn{2}{|c|}{$1.9 \pm 1.1$} & \multicolumn{2}{|c|}{$1.8 \pm 1.0$} & \multicolumn{2}{|c|}{$1.7 \pm 0.9$} & \multicolumn{2}{|c|}{$1.9 \pm 1.1$} \\
\hline \multicolumn{9}{|l|}{ Pregnancy order } \\
\hline 1 & 9,493 & 41.6 & 14 & 41.2 & 16,296 & 42.8 & 24 & 33.8 \\
\hline 2 or more & 13,316 & 58.4 & 50 & 58.8 & 21,784 & 57.2 & 47 & 66.2 \\
\hline Mean, S.D. & \multicolumn{2}{|c|}{$2.1 \pm 1.4$} & \multicolumn{2}{|c|}{$2.1 \pm 1.2$} & \multicolumn{2}{|c|}{$1.9 \pm 1.2$} & \multicolumn{2}{|c|}{$2.2 \pm 1.2$} \\
\hline Categorical & No. & $\%$ & No. & $\%$ & No. & $\%$ & No. & $\%$ \\
\hline Unmarried & 1,265 & 5.5 & 4 & 11.8 & 1,467 & 3.9 & 5 & 7.0 \\
\hline Employment status & & & & & & & & \\
\hline Professional & 1,969 & 8.6 & 8 & 23.5 & 4,399 & 11.6 & 24 & 33.8 \\
\hline Managerial & 5,083 & 22.3 & 14 & 41.2 & 10,249 & 26.9 & 16 & 22.5 \\
\hline Skilled worker & 6,493 & 28.5 & 8 & 23.5 & 11,886 & 31.2 & 22 & 31.0 \\
\hline Semiskilled worker & 4,196 & 18.4 & 1 & 2.9 & 6,159 & 16.2 & 2 & 2.8 \\
\hline Unskilled worker & 1,775 & 7.8 & 1 & 2.9 & 2,187 & 5.7 & 0 & 0.0 \\
\hline Housewife & 2,404 & 10.5 & 2 & 5.9 & 2,351 & 6.2 & 3 & 4.2 \\
\hline Others & 889 & 3.9 & 0 & 0.0 & 849 & 2.2 & 4 & 5.6 \\
\hline Pregnancy suppleme & & & & & & & & \\
\hline Iron & 14,721 & 64.5 & 21 & 61.8 & 26,722 & 70.2 & 49 & 69.0 \\
\hline Calcium & 1,798 & 7.9 & 5 & 14.7 & 3,570 & 9.4 & 13 & 18.3 \\
\hline Folic acid & 11,263 & 49.4 & 16 & 47.1 & 20,736 & 54.5 & 39 & 54.9 \\
\hline Vitamin B6 & 2,010 & 8.8 & 3 & 8.8 & 4,080 & 10.7 & 6 & 8.5 \\
\hline Vitamin D & 6,093 & 26.7 & 8 & 23.5 & 10,131 & 26.6 & 19 & 26.8 \\
\hline Vitamin C & 908 & 4.0 & 4 & 11.8 & 1,681 & 4.4 & 4 & 5.6 \\
\hline Vitamin E & 1,410 & 6.2 & 8 & 23.5 & 2,281 & 6.0 & 6 & 8.5 \\
\hline Multivitamin & 1,328 & 5.8 & 2 & 5.9 & 2,501 & 6.6 & 8 & 11.3 \\
\hline
\end{tabular}


Table 2. Incidence of pregnancy complications in women with or without leiomyoma in pregnancy (LP).

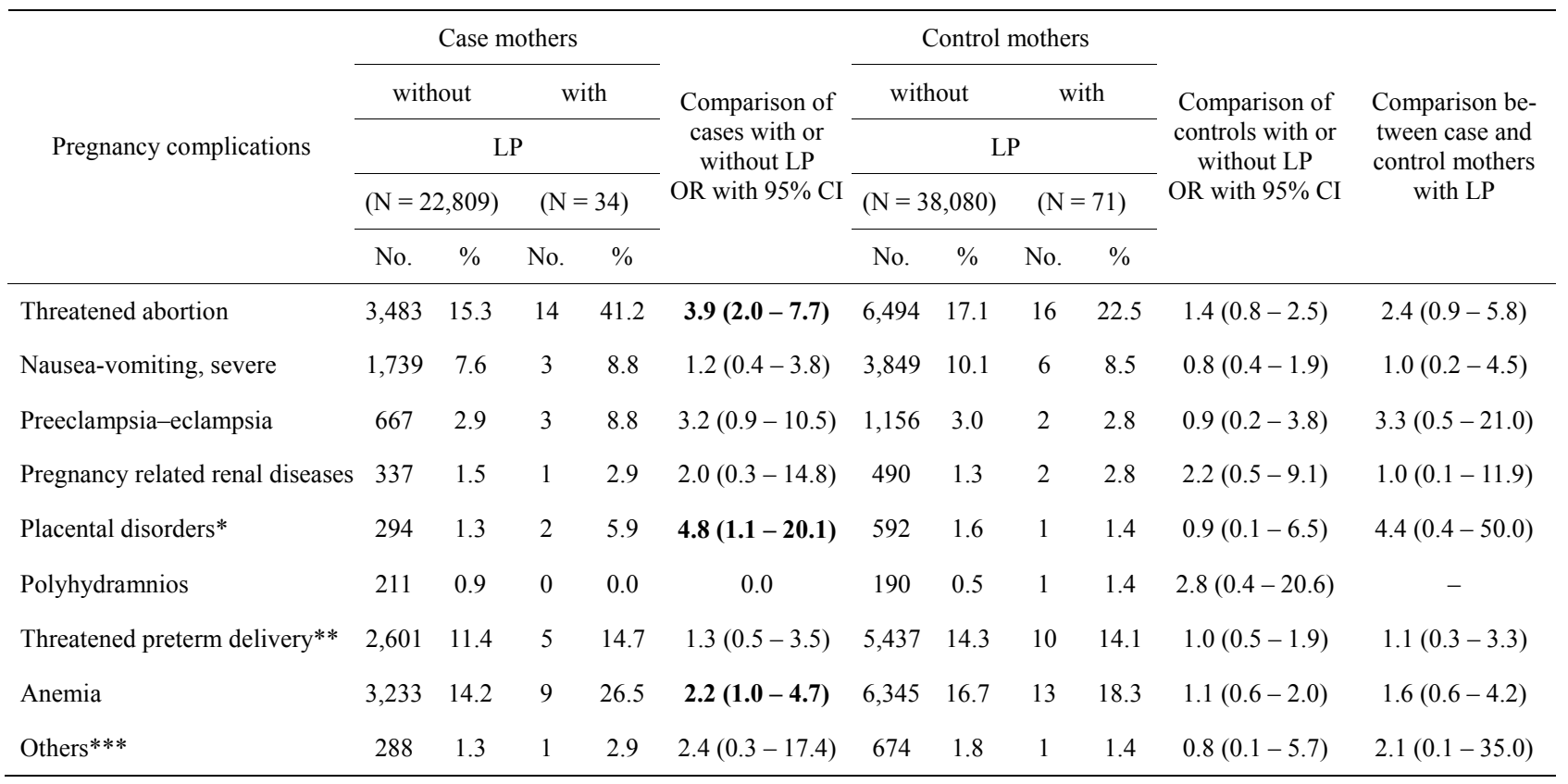

*incl. placenta praevia, premature separation of

**incl. cervical incompetence as well

***e.g. trauma, poisoning, blood isoimmunisation

Bold numbers show significant associations

Table 3. Birth outcomes of cases and controls born to mothers with or without leiomyoma in pregnancy (LP).

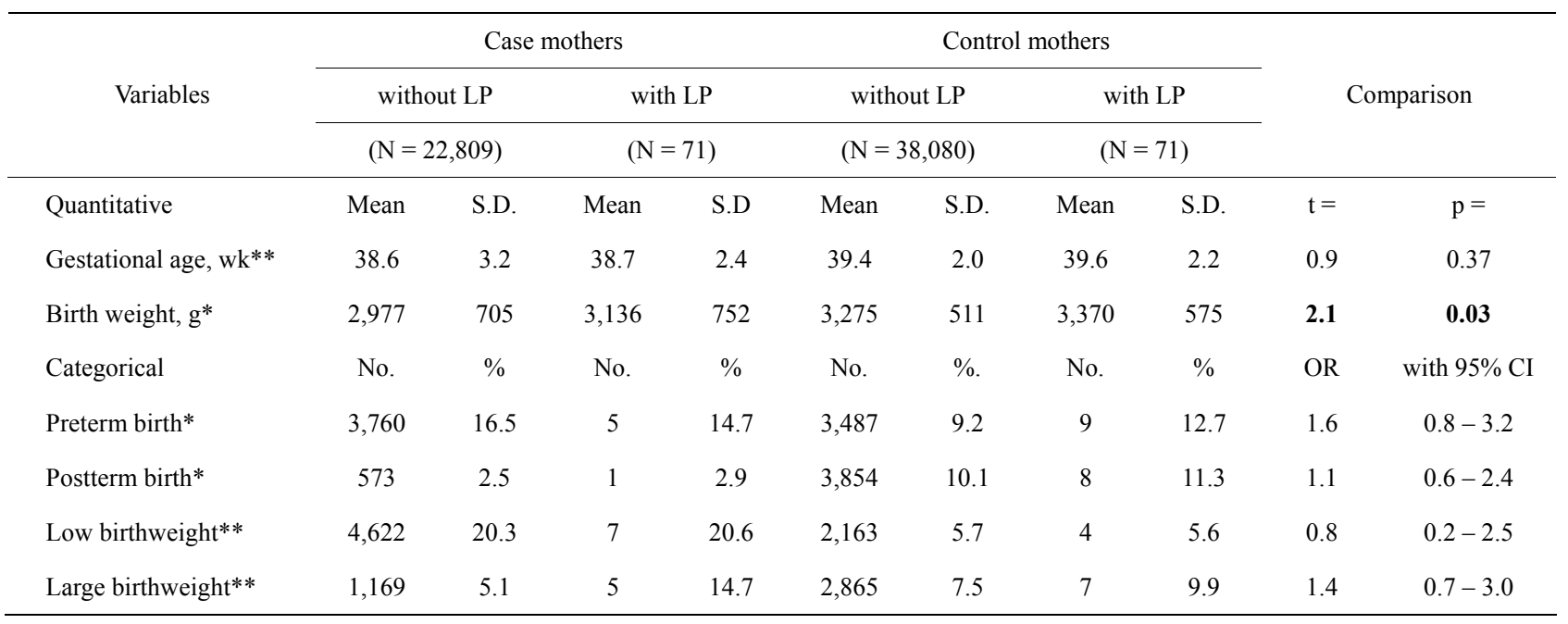

*adjusted for maternal age, birth order and maternal socio-economic status

**adjusted for maternal age, birth order, maternal socio-economic status and gestational age

Bold numbers show significant association

At the estimation of possible higher risk for CAs, the occurrence of LP during the entire pregnancy of mothers who had cases with different CAs was compared with the occurrence of LP in the mothers of all matched controls (Table 4). (We supposed that 4 case and 11 control mothers with the diagnosis of LP after the fourth gestational month might have some effect for the uterus during the critical period of most major CAs, i.e. during the second and third gestational month.) There was no higher risk for total CA and any CA group, including 
Table 4. Estimate the association between women with leimyoma in pregnancy (LP) and different CAs in their offspring using all matched controls as reference.

\begin{tabular}{|c|c|c|c|c|c|c|c|}
\hline \multirow{2}{*}{ Study groups } & \multirow{2}{*}{ Grand total No. } & \multicolumn{2}{|c|}{ Entire pregnancy } & \multicolumn{2}{|c|}{ Crude } & \multicolumn{2}{|c|}{ Adjusted } \\
\hline & & No. & $\%$ & OR & $95 \% \mathrm{CI}$ & OR & $95 \% \mathrm{CI}$ \\
\hline Controls & 38,151 & 71 & 0.2 & \multicolumn{2}{|c|}{ reference } & \multicolumn{2}{|c|}{ reference } \\
\hline \multicolumn{8}{|l|}{ Isolated CAs } \\
\hline Neural-tube defects & 1,203 & 2 & 0.2 & 0.9 & $0.2-3.6$ & 0.6 & $0.1-3.3$ \\
\hline Cleft lip \pm palate & 1,374 & 3 & 0.2 & 1.2 & $0.4-3.7$ & 0.8 & $0.2-3.4$ \\
\hline Cleft palate only & 601 & 2 & 0.3 & 1.8 & $0.4-7.3$ & 3.5 & $0.3-39.1$ \\
\hline Hypospadias & 3,038 & 9 & 0.3 & 1.6 & $0.8-3.2$ & 1.3 & $0.5-3.3$ \\
\hline Undescended testis & 2,051 & 3 & 0.1 & 0.8 & $0.2-2.5$ & 1.1 & $0.3-4.8$ \\
\hline Cardiovascular CAs & 4,479 & 6 & 0.1 & 0.7 & $0.3-1.7$ & 0.9 & $0.3-2.4$ \\
\hline Clubfoot & 2,424 & 4 & 0.2 & 0.9 & $0.3-2.4$ & 0.6 & $0.2-2.1$ \\
\hline Limb deficiencies & 548 & 3 & 0.5 & 3.0 & $0.9-9.4$ & 1.4 & $0.3-6.0$ \\
\hline Other isolated CAs & 5,776 & $2 * *$ & 0.0 & 0.2 & $0.0-0.8$ & 0.2 & $0.0-0.8$ \\
\hline Multiple CAs & 1,349 & 0 & 0.0 & 0.0 & $0.0-0.0$ & - & - \\
\hline Total & 22,843 & 34 & 0.1 & 0.8 & $0.5-1.2$ & 0.7 & $0.5-1.1$ \\
\hline
\end{tabular}

*ORs adjusted for maternal age and employment status, use of folic acid during pregnancy, and birth order

**torticollis, branchial cyst

clubfoot (i.e. typical manifestation of postural deformation due to fetal malposition).

\section{DISCUSSION}

We examined the possible association between LP and pregnancy complications, in addition birth outcomes. The previously found higher risk of threatened abortion and placental disorders particularly abruption placentae was found $[4-6,8,20]$, but this risk was significant only in the mothers of cases with CA, but not in the mothers of controls without CA in our study. Birth outcomes showed controversial pattern: the previously reported somewhat higher rate of preterm birth was confirmed (e.g. [9]), but only in controls without CA and this increase was not significant. On the other hand, there was a larger mean birth weight of cases and controls born to mothers with LP, but it does not associate with a lower risk of low birthweight. In fact cases had a higher rate of large birthweight. There was no higher risk of total and any CA group.

The secondary findings of the study confirmed the well-known fact that LP is more frequent in elder pregnant women (e.g. [8]). The advance maternal age may explain the higher prevalence of essential hypertension, haemorrhoids and constipation. Previously the higher rate of anaemia (mostly iron deficiency) in women with LP due to abnormal menstruation and haemorrhoids (frequently with bleeding) was not frequently mentioned. However, it is worth mentioning that these associations achieved the significant level only in case mothers thus this study suggests that case mothers with LP (i.e. having a malformed fetus) had a higher risk of pregnancy complications.

The higher mean maternal age did not associate with a higher mean birth order, and the difference between birth and pregnancy order was somewhat larger in women with LP likely due to the higher rate of previous miscarriages [21].

The high use of vitamin E (particularly in case mothers), human chorionic gonadotropin and hydroxyprogesterone may indicate some infertility problem in women affected with leiomyoma.

The prevalence of LP ranged from $0.1-3.9 \%$ in previous epidemiological studies $[4,6,20,22,23]$, while this prevalence was $0.15-0.19 \%$ in our study, i.e. near to the lower level of this range. A similar lower prevalence $(0.37 \%)$ was found in another population-based material [8]. Of course, the prevalence is determined by the age distribution of women and the diagnostic criteria, in addition underreporting might occur if a woman did not have a sonographic examination, or the diagnosis was 
not reported in the prenatal maternity logbook.

The unexpected finding of the study is the larger mean birth weight, and a somewhat higher rate of large birthweight newborns of pregnant women with LP. Coronado et al. [8] reported a higher rate of low birthweight newborns and prolonged labor. Our data were not appropriate to evaluate the latter, but newborns had larger birth weight. Women with LP had a better socioeconomic status but this confounder was considered at the calculation of adjusted mean birth weight. Obviously these pregnant women at high risk had also a special prenatal medical management but it did not associate with a higher level of folic acid supplementation. Thus further studies are needed to check the efficacy of recent medical management of women with LP and to explain some unexpected findings in this study.

The strengths of the HCCSCA are that is a population-based and large data set including 105 women with prospectively and medically recorded LP in prenatal maternity logbook, furthermore medically recorded gestational age at delivery and birth weight in an ethnically homogeneous Hungarian (Caucasian) population. Additional strengths include the matching of cases to controls without CAs; available data for potential confounders, and finally that the diagnosis of medically reported CAs was checked in the HCAR [11] and later modified, if necessary, on the basis of recent medical examination within the HCCSCA [10].

However, this data set also has limitations. 1) There is underreporting of LP in our data set. 2) The occurrence of previous surgical and other medical management in women with leiomyoma was not checked in validation studies, only the medically recorded data in the prenatal maternity logbook were evaluated 3) The size of LP was not recorded thus there was no chance to estimate the dose-effect relation. 4) The occurrence of previous miscarriages could be estimated only on the basis of difference of birth and pregnancy order in the data set of the HCCSCA, in addition the higher risk of women with LP was supported by the higher rate of vitamin $\mathrm{E}$ and hydroxyprogesterone treatment. 5) The lifestyle data were known only in the subsamples of pregnant women visited at home because previous validation study indicated the unreliability of maternal information regarding their smoking and drinking habit [24].

In conclusion, a higher occurrence of threatened abortion and placental disorders was found in the mothers of cases with CA, but not in the mothers of controls without CA. There was larger mean birth weight of babies born to mothers with LP and it associated with a higher rate of large birthweight in cases. A higher risk of CAs was not found among the offspring of pregnant women with LP. Thus the pregnancy of women with uterine leiomyoma does not to be discouraged if they wish to have babies, but they need specific and high medical care.

\section{REFERENCES}

[1] Stewart, E.A. (2001) Uterine fibroids. Lancet, 357(9752), 293-298.

[2] Ouyang, D. and Hill, J.A. (2002) Leiomyoma, pregnancy and pregnancy loss. Infertility and Reproductive Medicine Clinics of North America, 13, 325.

[3] Agdi, M. and Tulandi, T. (2008) Endoscopic management of uterine fibroids. Best Practice and Research Clinical Obstetrics and Gynacology, 22(4), 569-760.

[4] Katz, V.L., Dotters, D.J. and Droegemueller, W. (1989) Complications of uterine leiomyomas in pregnancy. $\mathrm{Ob}$ stetrics and Gynecology, 73(4), 593-596.

[5] Davis, J.L., Ray-Mazumder, S., Hobel, C.J., et al. (1990) Uterine leiomyomas in pregnancy: A prospective study. Obstetrics and Gynecology, 75(1), 41-44.

[6] Exacoustos, C. and Rosati, P. (1993) Ultrasound diagnosis of uterine myomas and complications in pregnancy. Obstet-rics and Gynecology, 82(1), 97-101.

[7] Vergani, P., Ghidini, A., Strobelt, N., et al. (1994) Do uterine leiomyomas influence pregnancy outcomes? American Journal of Perinatology, 11(5), 356-358.

[8] Coronado, G.D., Marshall, L.M. and Schwartz, S.M. (2000) Complications in pregnancy, labour, and delivery with uterine leiomyomas: a population-base study. $\mathrm{Ob}$ stetrics and Gynecology, 95(5), 767-769.

[9] Chen, Y-H., Lin, H-C., Chen, S.-F. and Lin, H.-C. (2009) Increased risk of preterm births among women with uterine leiomyoma: a nationwide population-based study. Human Reproduction, 24(12), 3049-3056.

[10] Czeizel, A.E., Rockenbauer, M., Siffel, C. and Varga, E. (2001) Description and mission evaluation of the Hungarian Case-Control Surveillance of congenital Abnormalities, 1980-1996. Teratology, 63(5), 176-185.

[11] Czeizel, A.E. (1997) The first 25 years of the Hungarian Congenital Abnormality Registry. Teratology, 55(5), 299305.

[12] Czeizel, A.E., Intődy, Z. and Modell, B. (1993) What proportion of congenital abnormalities can be prevented? British Medical Journal, 306(6880), 499-503.

[13] Czeizel, A.E., Petik, D. and Vargha, P. (2003) Validation studies of drug exposures in pregnant women. Pharmacoepidemiology and Drug Safety, 12(5), 409-416.

[14] Czeizel, A.E. and Vargha, P. (2004) Periconceptional folic acid/multivitamin supplementation and twin pregnancies. American Journal of Obstetrics and Gynecology, 191(3), 790-794.

[15] Czeizel, A.E. and Dudas, I. (1992) Prevention of the first occurrence of neural-tube defects by periconceptional vitamin supplementation. New England Journal of Medicine, 327(26), 1832-1835.

[16] Czeizel, A.E. (1996) Reduction of urinary tract and cardiovascular defects by periconceptional multivitamin supplementation. American Journal of Medical Genetics, 62(2), 179-183.

[17] Czeizel, A.E., Dobo, M. and Vargha, P. (2004) Hungarian 
cohort-controlled trial of periconceptional multivitamin supplementation shows reduction in certain congenital abnormalities. Birth Defects Research, 70(11), Part A, $853-861$.

[18] Botto, L.D., Olney, R.S. and Erickson, J.D. (2004) Vitamin supplements and the risk for congenital anomalies other than neural-tube defects. American Journal of Medical Genetics, 125C(1), 12-21.

[19] Puho, H.E., Métneki, J. and Czeizel, A.E. (2004) Maternal employment status and isolated orofacial clefts in Hungary. Cental European Journal of Public Health, 13(3), 144-148.

[20] Rice, J.P., Kay, H.H. and Mahony, B.S. (1989) The clinical significance of uterine leiomyoma in pregnancy. American Journal of Obstetrics and Gynecology, 160 (5
Pt 1), 1212-1216.

[21] Probst, A.M. and Hill, J.A. (2000) Anatomic factors associated with recurrent pregnancy loss. Seminars in Reproductive Medicine, 18(4), 341-350.

[22] Burton, C.A., Grimes, D.A. and March, C.M. (1989) Surgical management of leiomyomata in pregnancy. $\mathrm{Ob}$ stetrics and Gynecology, 74(5), 707-709.

[23] Hasan, F., Arumugam, K. and Sivanesaratman, V. (1991) Uterine leiomyomata in pregnancy. International Journal of Gynaecology and Obstetrics, 34(1), 45-48.

[24] Czeizel, A.E., Petik, D. and Puho, E. (2004) Smoking and alcohol drinking during pregnancy. The reliability of retrospective maternal self-reported information. Central European Journal of Public Health, 12(4), 179-183. 